\title{
Avaliação da qualidade de farinhas de sorgo preparadas por diferentes tratamentos e comparação com farinhas de milho
}

Evaluation of the quality of sorghum flours prepared by different treatments and comparison with corn flours

Evaluación de la calidad de las harinas de sorgo preparadas mediante diferentes tratamentos y comparación com las de maíz

\section{Resumo}

O sorgo é o quinto cereal mais produzido no mundo, porém ainda é utilizado quase que na totalidade para alimentação animal. Apresenta potencial muito grande para a alimentação humana por sua composição, por ser um cereal sem glúten e também por aspectos agronômicos. Por ter uma composição semelhante ao milho, esse trabalho teve como objetivo submeter esse cereal a tratamentos geralmente utilizados para grits de milho, a extrusão e a nixtamalização, avaliando as farinhas obtidas por esses tratamentos. Foram elaboradas 7 amostras: 4 somente utilizando sorgo como matéria-prima, 1 combinando com grits de milho, e 2 tendo como matéria-prima o grits de milho. Uma das amostras a partir de sorgo não sofreu nenhum tratamento, uma delas sofreu nixtamalização, enquanto as outras foram extrusadas. Todas as amostras foram avaliadas quanto a umidade, índice de absorção de água e de solubilidade, teor de fibras, proteínas e cor. Os resultados mostram o potencial que o sorgo pode ter na alimentação humana, com destaque para o alto teor de proteínas, maior do que em amostras contendo grits de milho, e maior após o processo de extrusão. Conclui-se que é possível produzir farinhas pré-gelatinizadas a partir de grãos de sorgo, com potencial para as indústrias de alimentos.

Palavras-chave: Extrusão; Nixtamalização; Pré-gelatinização; Grits.

\begin{abstract}
Sorghum is the fifth most produced cereal in the world, but it is still used almost entirely for animal feed. It presents a great potential for human food due to its composition, for being a gluten-free cereal, and also due to agronomic aspects. For having a composition similar to corn, this work aimed to submit this cereal to treatments generally used for corn grits, extrusion and nixtamalization, evaluating the flours obtained by these treatments. Seven samples were prepared: 4 using only sorghum as raw material, 1 combined with corn grits, and 2 with corn grits as raw material. One of the sorghum samples did not undergo any treatment, one of them underwent nixtamalization, while the others were extruded. All samples were evaluated for moisture, water absorption and solubility index, fiber, protein and color content. The results show the potential that sorghum can have in human food, highlighting the high protein content, higher than in samples containing corn grits, and higher after the extrusion process. It is concluded that it is possible to produce pre-gelatinized flours from sorghum grains, with potential for food industries.
\end{abstract}

Keywords: Extrusion; Nixtamalization; Pre-gelatinization; Grits. 


\begin{abstract}
Resumen
El sorgo es el quinto cereal más producido en el mundo, pero sigue utilizándose casi exclusivamente para la alimentación animal. Presenta un gran potencial para la alimentación humana por su composición, por ser un cereal sin gluten y también por aspectos agronómicos. Por tener una composición similar a la del maíz, este trabajo tuvo como objetivo someter este cereal a los tratamientos habitualmente utilizados para los grañones de maíz, extrusión y nixtamalización, evaluando las harinas obtenidas por estos tratamientos. Se elaboraron siete muestras: 4 utilizando sólo sorgo como materia prima, 1 combinándolo con sémola de maíz y 2 teniendo sémola de maíz como materia prima. Una de las muestras de sorgo no se sometió a ningún tratamiento, una de ellas se sometió a la nixtamalización, mientras que las demás fueron extrusionadas. En todas las muestras se evaluó la humedad, el índice de absorción de agua y solubilidad, el contenido de fibra, las proteínas y el color. Los resultados muestran el potencial que puede tener el sorgo en la alimentación humana, destacando el alto contenido en proteínas, mayor que en las muestras que contienen sémola de maíz, y mayor tras el proceso de extrusión. Se concluye que es posible producir harinas pregelatinizadas a partir de granos de sorgo, con potencial para las industrias alimentarias.
\end{abstract}

Palabras clave: Extrusión; Nixtamalización; Pregelatinización; Sémola.

\title{
1. Introdução
}

O sorgo é um cereal sem glúten, rico em carboidratos complexos, minerais e compostos bioativos (Davana e Revanna, 2021; Gomes, 2017; Martino, Cardoso, Moraes, Sant'Ana \& Queiroz, 2014). Apresenta-se muito propício à alimentação humana por apresentar potencial atividade antioxidante, antidiabética, antiobesidade, anti-inflamatória, entre outros benefícios como um alimento funcional à saúde humana (Queiroz, Menezes, Barros \& Martino, 2015; Rashwan, Yones, Karim, Taha \& Chen, 2021).

É o quinto cereal mais produzido no mundo todo, sendo mais popular na alimentação humana no continente africano (Awika, 2011; Pontieri et al., 2020). Awika (2011, p.9) estima que apenas 50\% da produção mundial de sorgo é utilizada para alimentação humana, o restante sendo destinada para alimentação animal. Os Estados Unidos é um dos maiores produtores mundiais, mas sua população praticamente não inclui o sorgo em sua dieta, destinando a produção à alimentação animal ou exportação (Awika, 2011). No Brasil praticamente toda a produção também é destinada à alimentação animal (Gomes, 2017).

Contudo, nos últimos anos o potencial do sorgo na saúde humana e na prevenção de doenças em geral vem ganhando destaque e diversos autores vem estudando sua aplicação em alimentos que já fazem parte da rotina de alimentação humana, como produtos de panificação, snacks, farinhas, cervejas e outras bebidas, entre outros (Martino et al, 2014; Pontieri et al., 2020). Além de nutricionalmente, a produção desse cereal tem diversas vantagens do ponto de vista agronômico, como menor custo de produção, possibilitando uma redução de custos posteriormente nas indústrias (Martino et al, 2014; Queiroz et al., 2015).

Sua composição é semelhante à do milho, possuindo em todas as variedades principalmente amido, por isso as tecnologias comumente utilizadas para esse podem ser aplicáveis a fim de explorar o potencial do sorgo como matéria-prima para a produção de outros produtos alimentícios (Martínez \& Pau, 1992; Queiroz et al., 2015).

O processo de extrusão muito empregado com produtos derivados do milho pode ser adequado para o sorgo, uma vez que há menor destruição de nutrientes e melhor digestibilidade (Gomes, 2017; Martínez \& Pau, 1992). Alimentos como salgadinhos, massas e macarrão são fabricados através da extrusão e como o sorgo é um cereal sem glúten, podem ser produzidos alimentos destinados à população celíaca ou com outras doenças autoimunes (Rashwan et al., 2021).

A nixtamalização é outro processo utilizado para melhorar a qualidade nutricional do milho, onde, com a presença de uma solução alcalina, busca-se facilitar a moagem, melhorar o sabor e o valor nutritivo (Brazil, Santos, Coura \& Minuzzo, 2020). Alguns estudos demonstram que, para o sorgo, esse processo pode ainda remover a presença de possíveis antinutrientes presentes (Rashwan et al., 2021).

Diante deste cenário, em que o sorgo é pouco utilizado na alimentação humana, mas com um enorme potencial, essa pesquisa teve como objetivo produzir farinhas à base de sorgo por meio de diferentes tratamentos, comumente utilizados em 
matérias-primas à base de milho, avaliando suas características físico-químicas como sendo promissor na aplicação em indústrias de alimentos.

\section{Metodologia}

\section{Material}

Os grãos sorgo utilizados, BM 709 da Biomatrix, foram doados por uma cooperativa do norte do Paraná (Cocamar Cooperativa Agroindustrial, Brasil). O grits de milho foi doado por uma empresa localizada em Maringá-PR (Nutrimilho, Brasil).

\section{Preparo das amostras}

Os grãos de sorgo foram devidamente limpos manualmente, para remoção de sujidades, e triturados em moinho de facas, formando a amostra denominada de S. A amostra S foi umidificada acrescentando-se $2 \%$ de água sobre seu peso (amostra S2) e também 40\% de água (amostra S40). A farinha também foi submetida ao processo de nixtamalização, onde uma solução de $0,3 \%$ de cal foi adicionada na proporção de $36 \%(\mathrm{~m} / \mathrm{v})$. Foi preparada uma amostra combinando $50 \%$ de massa do sorgo moído e 50\% de grits de milho, sendo adicionado 2\% de água sobre o peso total (amostra SM). Amostras de grits adicionando-se $2 \%$ e $40 \%$ de água também foram preparadas (M2 e M40, respectivamente).

Todas as amostras, com exceção da amostra $\mathrm{S}$, foram submetidas ao processo de extrusão no equipamento IMBRA RX50 (INBRAMAQ, Ribeirão Preto SP), com rosca única de $50 \mathrm{~mm}$ de diâmetro e $200 \mathrm{~mm}$ de comprimento, matriz com dois furos de $3 \mathrm{~mm}$ de diâmetro (Berwig et al, 2017). Posteriormente todos os tratamentos foram triturados novamente em moinho de facas, sendo armazenadas até o momento das análises.

\section{Análises físico-químicas}

A umidade das amostras foi determinada de acordo com metodologia AOAC (2005), por secagem em estufa a $105^{\circ} \mathrm{C}$. A cor das amostras foi medida através de dez medições para cada tratamento, por colorímetro digital CR-400 (Konica Minolta). Os resultados são expressos, no sistema CIELAB, em valores L*, a* e b*.

Os índices de absorção de água (IAA) e de solubilidade em água (ISA) foram determinados conforme metodologia descrita por Anderson et al. (1970). As amostras foram caracterizadas pelos teores de fibras (Cecchi, 2003) e proteínas via método de Kjeldahl (AOAC, 2005).

\section{Análise estatística}

Todas as análises, com exceção da análise de cor, foram realizadas em triplicata. Os resultados de todas as análises foram submetidos à análise de variância (ANOVA) com posterior análise das médias, comparadas pelo teste de Tukey ao nível de significância de 5\% através do software Assistat 7.7.

\section{Resultados e Discussão}

A Tabela 1 apresenta os resultados das análises de umidade, índice de absorção de água e solubilidade em água.

É possível observar que o teor de umidade foi maior na farinha de sorgo sem tratamentos (S), o que se justifica, porque as demais amostras sofreram o processo de extrusão, onde ocorre vaporização da água (Gomes, 2017). A umidade encontrada para S está de acordo com o que Dicko, Gruppen, Traoré, Voragen e Berkel (2006) apresentam como uma umidade aproximada do grão de sorgo (8-12\%). As amostras S2 e SM, com umidades semelhantes estatisticamente, apresentaram teor conforme Correia et al. (2020), que também avaliaram farinhas de sorgo produzidas a partir de extrusão. 
Tabela 1 - Resultados das análises de umidade, índice de absorção em água (IAA) e índice de solubilidade em água (ISA).

\begin{tabular}{cccc}
\hline Amostra & Umidade $(\%)$ & IAA & ISA \\
\hline S & $12,26^{\mathrm{a}} \pm 0,16$ & $2,39^{\mathrm{f}} \pm 0,01$ & $0,77^{\mathrm{e}} \pm 0,02$ \\
$\mathrm{~S} 2$ & $8,20^{\mathrm{b}} \pm 0,24$ & $4,68^{\mathrm{b}} \pm 0,01$ & $1,42^{\mathrm{cd}} \pm 0,14$ \\
$\mathrm{~S} 40$ & $4,98^{\mathrm{d}} \pm 0,17$ & $3,55^{\mathrm{c}} \pm 0,03$ & $0,84^{\mathrm{e}} \pm 0,02$ \\
SM & $8,18^{\mathrm{b}} \pm 0,24$ & $5,57^{\mathrm{a}} \pm 0,01$ & $1,93^{\mathrm{b}} \pm 0,03$ \\
SN & $3,90^{\mathrm{e}} \pm 0,09$ & $3,17^{\mathrm{d}} \pm 0,03$ & $1,64^{\mathrm{c}} \pm 0,01$ \\
M2 & $7,26^{\mathrm{c}} \pm 0,22$ & $5,53^{\mathrm{a}} \pm 0,01$ & $4,06^{\mathrm{a}} \pm 0,03$ \\
M40 & $3,13^{\mathrm{f}} \pm 0,14$ & $2,57^{\mathrm{e}} \pm 0,02$ & $1,26^{\mathrm{d}} \pm 0,03$ \\
\hline
\end{tabular}

Letras diferentes nas colunas indicam diferença significativa $(\mathrm{p}<0,05)$ entre os tratamentos. Fonte: Autores $(2021)$.

Dentre os tratamentos utilizando somente sorgo como matéria-prima, a amostra que passou pela nixtamalização foi a que apresentou menor teor de umidade. Essa diminuição na umidade que ocorreu com todos os tipos de tratamento é muito favorável para farinhas, pensando em seu armazenamento e aumento de shelf-life (Rodrigues, Resende, Silva \& Junior, 2020). O teor de umidade em farinha de trigo, segundo legislação no Brasil, pode ser de no máximo 15\% e em canjica de milho, de no máximo 13\%, dessa forma os valores encontrados nas amostras estão dentro do aceitável para farinhas (Brasil, 2005; Brasil, 1989).

Os índices de absorção e de solubilidade em água são importantes para caracterizar farinhas extrusadas, por isso é possível verificar que a amostra S, que não sofreu processo de extrusão, foi a que apresentou menores índices. O tratamento térmico aumenta a capacidade de absorção de água, gelatinizando o amido presente nas amostras, justificando o aumento dos índices em todas as outras amostras, que diferente da S, foram extrusadas (da Silva, Ascheri, Ascheri \& de Carvalho, 2008). Observou-se com destaque as amostras SM e M2, que tanto para o IAA, quanto para o ISA, apresentaram os maiores valores. O ISA verifica o grau de intensidade de tratamentos térmicos, uma vez que se relaciona à quantidade de sólidos solúveis presentes (Moura et al., 2011). Já o IAA indica a integralidade da estrutura amilácea após a extrusão, refletindo a capacidade do grânulo de amido absorver água (Moura et al., 2011; da Silva et al., 2008).

A Tabela 2 apresenta os teores de fibras e proteínas das amostras analisadas.

As amostras produzidas com sorgo apresentaram maiores teores de fibras e proteínas do que aquelas a partir de grits de milho ou de uma combinação dos dois cereais. A adição de grits de milho na amostra SM diminuiu tanto o teor de proteínas como de fibras, assim como as amostras M2 e M40 apresentaram menores teores por serem produzidas apenas com grits.

Segundo alguns autores, o teor de proteínas nos grãos de sorgo varia entre 7,3 e 15,6\%, enquanto o teor de fibras, entre 1,2 e 6,6\%, de acordo com o cultivar (Dicko et al., 2006; Martino et al., 2015). Os resultados encontrados para todas as amostras produzidas com sorgo apresentaram teores nessa faixa segundo a literatura. 
Tabela 2 - Resultados do teor de fibras e de proteínas.

\begin{tabular}{ccc}
\hline Amostra & Fibras (\%) & Proteínas (\%) \\
\hline S & $3,19^{\mathrm{a}} \pm 0,06$ & $9,05^{\mathrm{b}} \pm 0,07$ \\
S2 & $2,07^{\mathrm{c}} \pm 0,03$ & $9,26^{\mathrm{ab}} \pm 0,06$ \\
S40 & $2,48^{\mathrm{b}} \pm 0,11$ & $9,51^{\mathrm{ab}} \pm 0,13$ \\
SM & $1,82^{\mathrm{cd}} \pm 0,07$ & $8,10^{\mathrm{c}} \pm 0,14$ \\
SN & $2,00^{\mathrm{c}} \pm 0,07$ & $9,68^{\mathrm{a}} \pm 0,25$ \\
M2 & $1,63^{\mathrm{d}} \pm 0,06$ & $6,54^{\mathrm{d}} \pm 0,08$ \\
M40 & $1,05^{\mathrm{e}} \pm 0,03$ & $7,01^{\mathrm{d}} \pm 0,08$ \\
\hline
\end{tabular}

Letras diferentes nas colunas indicam diferença significativa $(\mathrm{p}<0,05)$ entre os tratamentos. Fonte: Autores $(2021)$.

Algumas propriedades da farinha de sorgo podem ser destacadas usando diferentes tratamentos, como, por exemplo, a extrusão (Correira et al., 2020; Dube, Xu \& Zhao, 2021; Rashwan et al., 2021). Isso aconteceu para proteínas, mas não para fibras. O processo de nixtamalização não se apresentou vantajoso para o destaque de proteínas e fibras, uma vez que a amostra SN não apresentou diferenças significativas com as amostras S2 e S40 para o teor de proteínas, e para o teor de fibras, não apresentou diferenças significativas com a amostra S2, e apresentando menor teor do que a amostra S40. Somado a isso, o processo de extrusão se destaca sobre a nixtamalização, por ser um procedimento relativamente simples, sem uso de reagentes e considerado limpo (Bombo, 2006).

A composição das farinhas de cereais pode variar de acordo com o genótipo da planta, condições climáticas e outros fatores (Queiroz et al., 2015). Para a farinha de trigo, o teor de proteínas em geral varia de 8 a 16\% (Scheuer, de Francisco, de Miranda \& Limberger, 2011), já para snacks extrusados a partir de grits de milho, Bombo (2006) encontrou um teor de proteínas de 7,45\%. Então ao se comparar com os teores encontrados nessa pesquisa, o sorgo mostra-se como uma ótima substituição em alimentos, não prejudicando os aspectos nutricionais. Ainda, há a vantagem de ser um cereal sem glúten, podendo ser aplicado em alimentos direcionados ao público celíaco ou que busca dietas restritivas de glúten, e de possuir maior teor de fibras.

A Tabela 3 apresenta os resultados para análise de cor das farinhas. É possível observar que os diferentes tratamentos fizeram com que as farinhas resultassem em diferentes colorações, mesmo quando utilizando apenas o sorgo como matériaprima. O sorgo pode ser fonte natural de corantes para a indústria de alimentos (Queiroz et al., 2015). Essa variação encontrada na análise realizada poderia oferecer uma maior variedade de tons à indústria de corantes, dependendo do tratamento empregado no processamento dos grãos de sorgo.

A variedade de sorgo utilizada nesta pesquisa foi de um grão mais escuro. Essa influência na cor pode ser percebida observando os valores de $\mathrm{L}^{*}$, que indicam a luminosidade nas amostras. Ou seja, os valores de $\mathrm{L}^{*} \mathrm{em}$ amostras contendo grits de milho foram maiores. Os valores de b* também foram maiores nessas amostras, pois esse parâmetro indica a intensidade da cor amarela, característica do grits de milho. O parâmetro a*, por sua vez, indica a escala de cor vermelha. Os valores foram maiores para as amostras a partir de sorgo, tendo relação direta com a cor do grão in natura. 
Tabela 3 - Resultados da análise de cor.

\begin{tabular}{cccc}
\hline \multirow{2}{*}{ Amostra } & \multicolumn{3}{c}{ Cor } \\
\cline { 2 - 4 } & $\mathbf{L}^{*}$ & $\mathbf{a}^{*}$ & $\mathbf{b}^{*}$ \\
\hline $\mathrm{S}$ & $62,88^{\mathrm{d}} \pm 1,61$ & $7,65^{\mathrm{c}} \pm 0,95$ & $16,30^{\mathrm{e}} \pm 0,76$ \\
S2 & $60,44^{\mathrm{e}} \pm 0,85$ & $8,38^{\mathrm{b}} \pm 0,15$ & $16,25^{\mathrm{e}} \pm 0,28$ \\
S40 & $50,64^{\mathrm{g}} \pm 1,85$ & $9,15^{\mathrm{a}} \pm 0,48$ & $17,96^{\mathrm{d}} \pm 0,84$ \\
SM & $69,98^{\mathrm{c}} \pm 0,98$ & $3,81^{\mathrm{d}} \pm 0,36$ & $29,51^{\mathrm{c}} \pm 0,85$ \\
SN & $53,41^{\mathrm{f}} \pm 2,40$ & $7,81^{\mathrm{bc}} \pm 0,28$ & $17,11^{\mathrm{de} \pm 0,57}$ \\
M2 & $84,77^{\mathrm{a}} \pm 1,76$ & $-6,74^{\mathrm{f}} \pm 0,16$ & $44,86^{\mathrm{b}} \pm 1,44$ \\
M40 & $77,32^{\mathrm{b}} \pm 1,36$ & $-0,32^{\mathrm{e}} \pm 0,59$ & $48,39^{\mathrm{a}} \pm 2,08$ \\
\hline
\end{tabular}

Letras diferentes nas colunas indicam diferença significativa $(\mathrm{p}<0,05)$ entre os tratamentos. Fonte: Autores $(2021)$.

No momento de aplicar essas farinhas de sorgo em produtos alimentícios esse tom mais escuro pode ser benéfico na aceitação dos consumidores, uma vez que alimentos integrais e mais saudáveis são muitas vezes associados pela população à essa característica visual (Martino et al., 2015).

\section{Conclusão}

É possível produzir farinhas pré-gelatinizadas a partir de grãos de sorgo, com potencial para o mercado de alimentos. Com o processo de extrusão, foi possível observar que ocorrem mudanças nos componentes do grão de sorgo e do grits de milho, pré-gelatinizando o amido presente, resultando em diminuição da umidade, aumento do IAA e ISA e mudanças de cor. A nixtamalização não apresentou diferencial sobre a extrusão, uma vez que a farinha que sofreu esse processo não apresentou resultados que se destacaram em comparação às outras amostras.

$\mathrm{O}$ teor de proteínas foi mais elevado nas amostras em que apenas sorgo foi utilizado como matéria-prima, o que confirma seu potencial como alimento humano. Estudos futuros devem avaliar a aplicação dessas farinhas em alimentos como produtos panificados, avaliando sua aceitação pelos consumidores.

Por ser um cereal sem glúten, o sorgo se apresenta como uma alternativa muito viável para as indústrias de alimentos. Dependendo do tratamento térmico empregado no processamento, o sorgo pode perder algumas de suas vitaminas e minerais, mas também antinutrientes, por isso trabalhos futuros deveriam investigar também a presença desses compostos nas farinhas de sorgo, avaliando a influência dos diferentes processos empregados.

\section{Referências}

Anderson, R. A., Conway, H. F., \& Peplinski, A. J. (1970). Gelatinization of corn grits by rool and extrusion cooking. Cereal Science Today, 14(1), 4-12.

AOAC - Association of Official Analytical Chemists (2005). Official methods of analysis of the AOAC International, (17a ed.) Gaitherburg.

Awika, J. M. (2011). Major cereal grains production and use around the world. In Advances in cereal science: implications to food processing and health promotion (pp. 1-13). American Chemical Society.

Berwig, K. P., Marques, D. R., Silva, D. M. B., Mendes, M. P., Raniero, G. Z., Monteiro, C. C. F., \& Monteiro, A. R. G. (2017). Texture on extruded snack: correlation between instrumental and sensory analysis. Chemical Engineering Transactions, 57, 1723-1728.

Bombo, A. J. (2006). Obtenção e caracterização nutricional de snacks de milho (Zea mays L.) e linhaça (Linum usitatissimum L.) (Dissertação de mestrado). Universidade de São Paulo, Faculdade de Saúde Pública, São Paulo, SP, Brasil.

Brazil, I. M., Santos, F. P., Coura, C. P., \& Minuzzo, D. A. (2020). Cultura alimentar venezuelana: culinária criolla e sua associação com dados de consumo da encuesta nacional de presupuestos familiares 2008-2009. Revista de Alimentação e Cultura das Américas, 1(1), 21-43.

Cecchi, H. M. (2003). Fundamentos teóricos e práticos em análise de alimentos: UNICAMP. 
Correia, V. T. V., D’Angelis, D. F., Rodrigues, C. G., Amante, P. R., Queiroz, V. A. V., Ferreira, A. A., \& Fante, A. C. (2020). Caracterização físico-química e tecnológica de farinha extrusada de sorgo do genótipo BRS 305. Research, Society and Development, 9(8), e115984963.

da Silva, E. M. M., Ascheri, J. L. R., Ascheri, D. P. R., \& de Carvalho, L. M. J. (2008). Efeito dos parâmetros de extrusão nas características de viscosidade de pasta e índice de absorção de água de macarrões pré-cozidos elaborados a partir de farinha mista de arroz integral e milho obtidos por extrusão. Embrapa Agroindústria de Alimentos-Artigo em periódico indexado (ALICE).

Davana, T. V., \& Revanna, M. L. (2021). Development of beer from malted sorghum (Sorghum bicolor). International Journal of Chemical Studies, 9(1), 330334.

Dicko, M. H., Gruppen, H., Traoré, A. S., Voragen, A. G. J., \& Berkel, W. J. H. (2006). Sorghum grain as human food in Africa: relevance of content of starch and amylase activities. African Journal of Biotechnology, 5(5), 384-395.

Gomes, A. C. G. (2017). Farinha pré-gelatinizada de quirera de arroz e de grãos de sorgo e sua aplicabilidade na elaboração de sopa instantânea (Dissertação de mestrado). Instituto Federal de Educação, Ciência e Tecnologia Goiano, Rio Verde, GO, Brasil.

Martínez, B. F., \& Pau, C. L. (1992). Extrusión de sorgo integral y decorticado. Agronomia Mesoamericana, 3, 40-44.

Martino, H. S. D., Cardoso, L. D. M., Moraes, É. A., Sant'Ana, H. M. P., \& Queiroz, V. A. V. (2014). Por que utilizar o sorgo na alimentação humana? Embrapa Milho e Sorgo-Capítulo em livro científico (ALICE).

Ministério da Agricultura, Pecuária e Abastecimento, Instrução Normativa 8/2005 (2005). Regulamento técnico de identidade e qualidade de farinha de trigo. Diário Oficial da União. Brasília.

Ministério da Agricultura, Pecuária e Abastecimento, Portaria 109-1989 (1989). Norma de identidade, qualidade, apresentação e embalagem da canjica de milho. Diário Oficial da União. Brasília.

Moura, L. D. M., Ascheri, J. L. R., Silva, C. D. O., Moro, T. D., de Carvalho, J. L. V., \& Nutti, M. R. (2011). Propriedades de absorção e solubilização de extrudados de farinha mista de feijão, milho e arroz biofortificados. In Embrapa Agroindústria de Alimentos-Artigo em anais de congresso (ALICE). In: Reunião De Biofortificação No Brasil, 4., 2011, Teresina. Palestras e resumos: Embrapa Agroindústria de Alimentos; Teresina: Embrapa Meio-Norte, 2011. 1 CD-ROM.

Pontieri, P., Troisi, J., Romano, R., Pizzolante, G., Bean, S. R., Tilley, M., Motto, M., Aletta, M., Giudice, F. D., Sicardi, M., Alifano, P., \& Giudice, L. D. (2020). Nutritional composition of a selected White food-grade waxy sorghum variety grown in Mediterranear environment. Australian Journal of Crop Science, 14(9), 1525-1532.

Queiroz, V. A. V., de Menezes, C. B., de Barros, F. A. R., \& Martino, H. S. D. (2015). O sorgo na alimentação humana. Embrapa Milho e Sorgo-Capítulo em livro científico (ALICE).

Rashwan, A. K., Yones, H. A., Karim, N., Taha, E. M., \& Chen, W. (2021). Potencial processing technologies for developing sorghum-based food products: An update and comprehensive review. Trends in Food Science \& Tecnology, 110, 168-182.

Rodrigues, G. B., Resende, O., Silva, L. C. M., \& Junior, W. N. F. (2020). Qualidade fisiológica das sementes de sorgo granífero durante o armazenamento. Research, Society and Development, 9(6), e27963152.

Scheuer, P. M., de Francisco, A., de Miranda, M. Z., \& Limberger, V. M. (2011). Trigo: características e utilização na panificação. Revista Brasileira de Produtos Agroindustriais, 13(2), 211-222. 